\title{
Anatomic Single Bundle Anterior Cruciate Ligament Reconstruction by the Two Anteromedial Portal Method: The Comparison of Transportal and Transtibial Techniques
}

\author{
Myung-Ku Kim, MD, PhD, Byung-Cheol Lee, MD and Joo-Hyun Park, MD \\ Department of Orthopaedic Surgery, Inha University Hospital, Inha University College of Medicine, Incheon, Korea
}

\begin{abstract}
Purpose: The purpose of this study was to report the results of anatomic single bundle anterior cruciate ligament (ACL) reconstruction by the two anteromedial portal method.

Materials and Methods: We evaluated the clinical results in 33 patients with ACL rupture who were treated by anatomic ACL reconstruction using the two anteromedial portal technique. The control group included 33 patients with ACL rupture who were treated with the conventional transtibial non-anatomic method. We performed an objective instability test, both preoperatively and at the final follow-up. We also compared the clinical results of both groups using International Knee Documentation Committee and Lysholm scores as a subjective test.

Results: At the final follow up, the study showed that in the control group, the Lachman test was negative in 27 cases (81.8\%), the pivot shift test was negative in 26 cases (78.8\%), and the average anterior translocation was $3.1 \mathrm{~mm}$ on a KT-2000 arthrometer. In the group of patients who underwent anatomic reconstruction by the two anteromedial portal method, the Lachman test was negative in 28 cases (84.8\%), the pivot shift test was negative in 30 cases (90.9\%), and the average anterior translocation was $2.8 \mathrm{~mm}$ on the KT-2000 arthrometer. Results in the pivot shift showed statistically significant improvement compared to the control group.

Conclusions: Anatomic ACL reconstruction by two anteromedial portals is an effective surgical technique that restores the rotational stability with excellent clinical results.
\end{abstract}

Key words: Anterior cruciate ligament reconstruction, Far anteromedial excavation, Rotational stability.

\section{Introduction}

Single-bundle anterior cruciate ligament (ACL) reconstruction

Received April 28, 2011; Revised (1st) May 31, 2011; (2nd) July 19, 2011; (3rd) September 7, 2011; Accepted September 8, 2011.

Correspondence to: Myung-Ku Kim, MD, PhD.

Department of Orthopaedic Surgery, Inha University Hospital, Inha University College of Medicine, Sinheung-dong 3-ga, Jung-gu, Incheon 400-711, Korea.

Tel: +82-32-890-3662, Fax: +82-32-890-3047

Email: m9kim@inha.ac.kr

*This study was supported by Inha University research grants.

This is an Open Access article distributed under the terms of the Creative Common Attribution Non-Commercial License (http://creativecommons.org/licenses/by-nc/3.0/) which permits unrestricted non-commercial use, distribution, and reproduction in any medium, provided the original work is properly cited.

Copyright ๑ 2011. KOREAN KNEE SOCIETY

www.jksrr.org is a common procedure that has success rates of 83 to $95 \%$. However, some biomechanical studies have shown that the procedure results in lower success rates than double-bundle ACL reconstruction ${ }^{1-3)}$. This can be attributed to the fact that single-bundle ACL reconstruction is not sufficient for complete restoration of the complex function of the anteromedial bundle, and especially of the posterolateral bundle ${ }^{4,5)}$. In addition, conventional single-bundle ACL reconstruction using an 11- or 1-o'clock femoral tunnel has been regarded as effective in restoring anteroposterior stability, but not rotational stability, because ACL ligament obliquity is increased after the procedure $^{6-8)}$.

Recently, the obliquity of a reconstructed ACL ligament has been reported as an important factor for rotational stability and long-term maintenance of knee function. Accordingly, some authors introduced anatomic reconstruction techniques designed to overcome disadvantages of the conventional nonanatomic reconstruction, including the ACL graft obliquity. 


\section{Kim et al. Anatomic Single Bundle Anterior Cruciate Ligament Reconstruction}

Conventional transtibial femoral tunnel drilling cannot be helpful for anatomic reconstruction, because it is difficult to place a tunnel at the center of the ACL attachment on the femur when the starting point is dependent on the site of the tibial tunnel. Harner and Poehling ${ }^{9)}$ used the anteromedial portal for femoral tunnel placement, but their method using the conventional anteromedial and anterolateral portals has been associated with poor visualization and cortical bone destruction.

In this study, we retrospectively evaluated the results of our new single-bundle ACL reconstruction technique using 3 portals by adding a far anteromedial portal to the frequently used 2 portals. We then compared our results with those of conventional ACL reconstruction using the transtibial femoral tunnel drilling technique.

\section{Materials and Methods}

\section{Materials}

The experimental group consisted of 33 patients with $\geq 12$ months of follow-up who had undergone anatomic singlebundle ACL reconstruction using 2 anteromedial portals and one anterolateral portal between September 2008 and January 2010 at our institution. The control group consisted of 33 patients who were similar in age to those in the experimental group and with $\geq 12$ months of follow-up who had undergone a conventional non-anatomic single-bundle ACL reconstruction

Table 1. Patient Demographics

\begin{tabular}{lcc}
\hline Patient demographics & Trans tibia & Far anteromedial \\
\hline Mean age & $30.3(19-51)$ & $29.8(17-58)$ \\
Sex (male/female) & $23 / 10$ & $25 / 8$ \\
Allograft/autograft & $27 / 6$ & $26 / 7$ \\
Mean follow-up (mo) & $32(12-55)$ & $20.8(12-29)$
\end{tabular}

$\mathrm{p}$-value $>0.05$. using transtibial femoral tunnel drilling between January 2003 and December 2006. The exclusion criteria were as follows: 1) an injury in the contralateral knee; and 2) an injury to the posterior cruciate ligament or medial/lateral collateral ligament that could affect the joint stability of the ACL-injured knee. In the control group, there were 10 females and 23 males, with a mean age of 30.3 years (range, 19 to 51 years). The affected side was the right side in 15 patients and left side in 18 patients. The surgery was done using a bone-patellar tendon-bone allograft in 27 patients and a bone-patellar tendon-bone autograft in 6 patients. The mean follow-up period was 32 months (range, 12 to 55 months). In the experimental group, there were 8 females and 25 males with a mean age of 29.8 years (range, 17 to 58 years). The affected side was the right side in 19 patients and the left side in 14 patients. The surgery was done using a bone-patellar tendonbone allograft in 26 cases and a bone-patellar tendon-bone autograft in 7 cases. The mean follow-up period was 20.8 months (range, 12 to 29 months) (Table 1 ).

\section{Surgical Technique}

The surgery was performed by a single surgeon for all patients. The ACL ruptures were confirmed by diagnostic arthroscopy. In the control group, the femoral tunnel was drilled using a transtibial technique. In the experimental group, our method using 2 anteromedial portals was performed for femoral tunnel placement. A standard anteromedial portal was created adjacent to the patellar tendon. Another anteromedial portal (far anteromedial portal) was established as distant as possible from the previous portal $(-2 \mathrm{~cm}$ from the medial border of the patellar tendon), using a needle at a site that allows for the use of a reamer without damaging the medial femoral condyle; thus avoiding complications that can be caused by interference with a reamer or an arthroscope (Fig. 1). A standard anterolateral portal was created. This method allows for arthroscopic visualization of the medial wall of the lateral femoral condyle through the standard
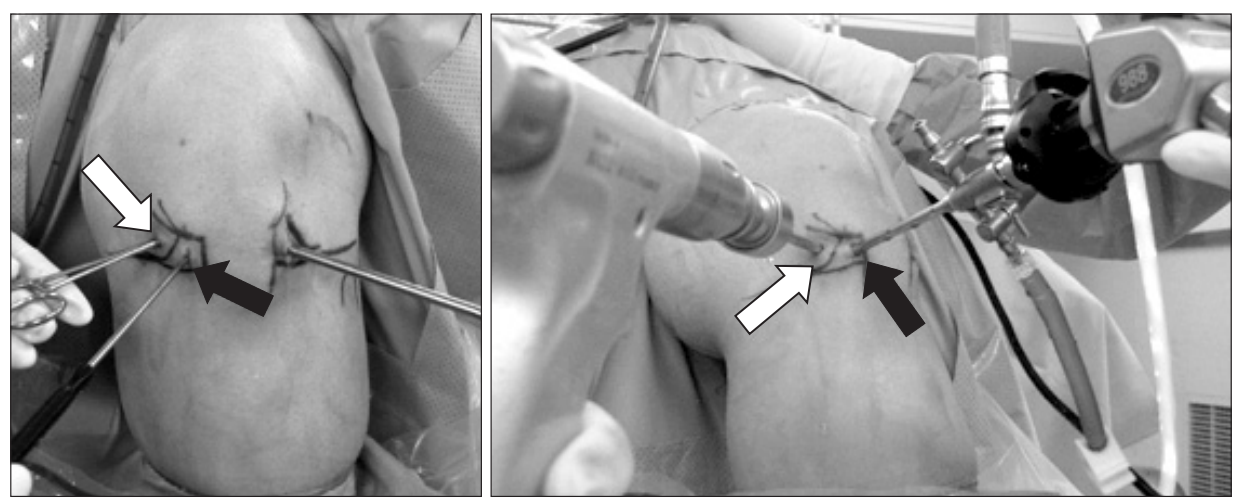

Fig. 1. Standard anteromedial portal (black arrow) and far anteromedial portal (white arrow). Far anteromedial (AM) portal is made slightly medial to the standard AM portal. 
anteromedial portal with the knee in hyperflexion; hence, the anatomic ACL insertion site on the femur and the posterior cortical bone can be located with ease (Fig. 2). A guide pin was placed at the center of the ACL insertion site on the femur through the far anteromedial portal and a femoral tunnel was created using a 10-mm reamer. A tibial tunnel was drilled with a guide pin placed at the center of the ACL insertion site on the tibia, taking care to preserve the remaining ACL tissue as much as possible (Fig. 3). Then, a graft was passed through the femoral tunnel and a bio-absorbable interference screw (BioScrew poly L-lactic acid, Linvatec Co., Largo, FL, USA) that was inserted through the far anteromedial portal was used for femoral fixation. A hybrid technique was used for tibial fixation of the graft.

\section{Methods}

For the assessment of knee stability, anterior tibial translation was evaluated using the Lachman test and a KT-2000 arthrometer, and rotational stability was evaluated using the pivot shift test.

For clinical assessment, the Lysholm score system and the International Knee Documentation Committee (IKDC) knee score system were used.

Femoral tunnel obliquity was measured on the knee tunnel view obtained postoperatively by aligning the X-ray beam parallel to the articular surface with the patient in the prone position and the knee maintained at $60^{\circ}$ of flexion using an angle block. The femoral tunnel obliquity was measured as the angle between a line parallel to the articular surface and the femoral tunnel. For
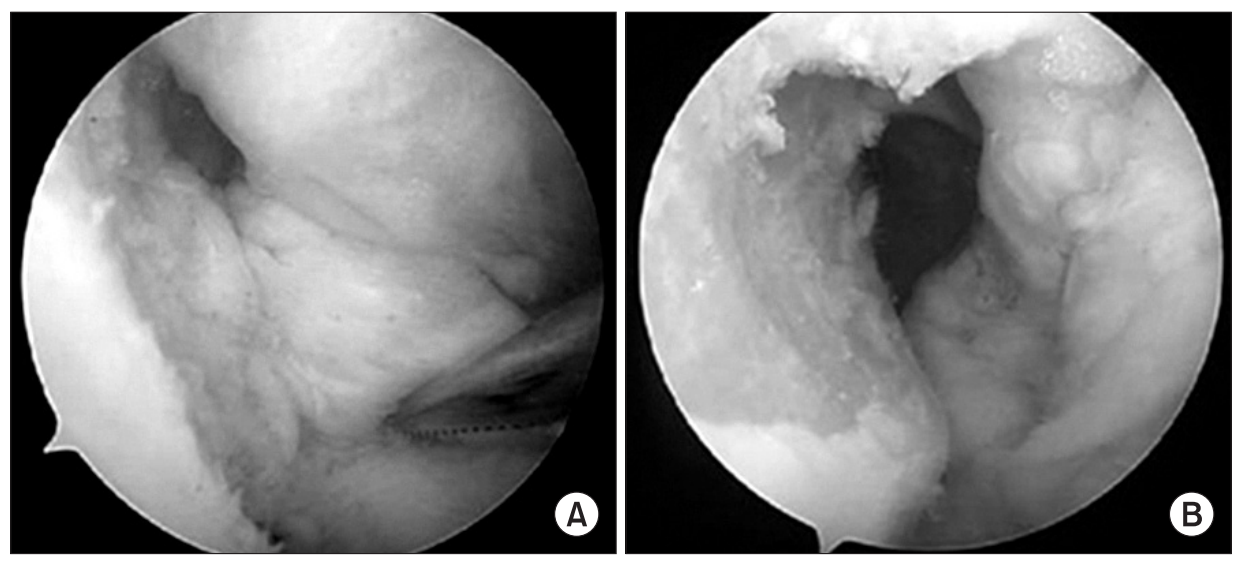

Fig. 2. Arthroscopic view through the standard anterolateral portal (A) and anteromedial portal (B). Anteromedial portal provides excellent arthroscopic view of medial wall of femur lateral condyle.
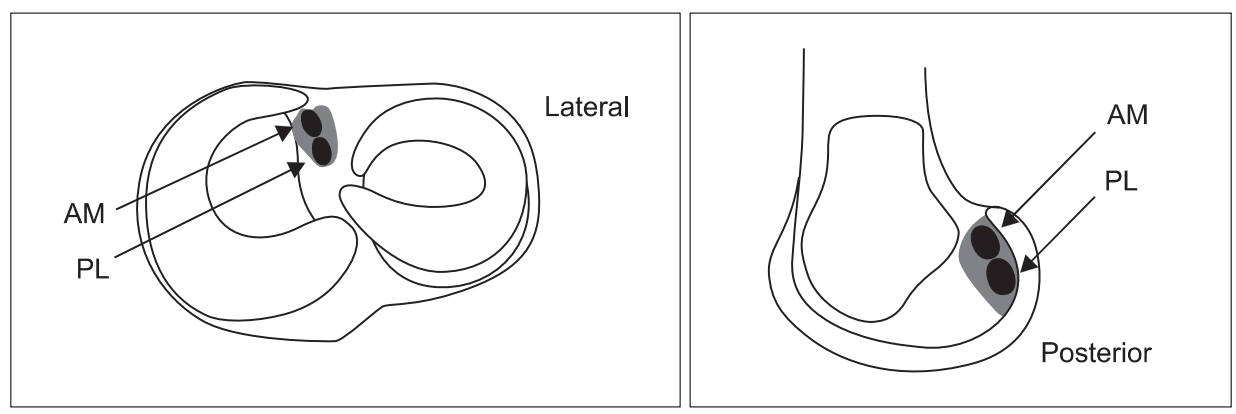

Fig. 3. Footprint of anteromedial (AM) and posterolateral (PL) bundles. The tunnel is made center of footprint of anteromedial and posterolateral bundles.

Table 2. Lachman Test

\begin{tabular}{lcc}
\hline Grade of Lachman test & Trans tibia & Far anteromedial \\
\hline 0 & $27(81.8)$ & $28(84.8)$ \\
I & $3(9.1)$ & $4(12.1)$ \\
II & $2(6.1)$ & $1(3.0)$ \\
III & $1(3.0)$ & $0(0.0)$ \\
\hline
\end{tabular}

p-value $>0.05$.

Values are presented as number (\%).
Table 3. KT-2000 Arthrometer

\begin{tabular}{lcc}
\hline KT-2000 $(\mathrm{mm})$ & Trans tibia & Far anteromedial \\
\hline$<3$ & $23(69.6)$ & $25(75.8)$ \\
$3-5$ & $7(21.2)$ & $6(18.2)$ \\
$>5$ & $3(9.0)$ & $2(6.0)$ \\
Average & 3.1 & 2.8 \\
\hline
\end{tabular}

p-value $>0.05$.

Values are presented as number (\%). 


\section{Kim et al. Anatomic Single Bundle Anterior Cruciate Ligament Reconstruction}

Table 4. Pivot Shift Test

\begin{tabular}{lcc}
\hline Grade of pivot shift test & Trans tibia & Far anteromedial \\
\hline 0 & $26(78.8)$ & $30(90.9)$ \\
I & $5(15.2)$ & $2(6.0)$ \\
II & $2(6.0)$ & $1(3.0)$ \\
III & $0(0.0)$ & $0(0.0)$
\end{tabular}

p-value $=0.04$.

Values are presented as number (\%).

Table 5. Lysholm Score

\begin{tabular}{lcc}
\hline Lysholm score & Trans tibia & Far anteromedial \\
\hline 5-100 (excellent) & $16(48.5)$ & $19(57.6)$ \\
$70-84$ (good) & $10(30.3)$ & $12(36.4)$ \\
$55-69$ (fair) & $4(12.1)$ & $1(3.0)$ \\
$0-54$ (poor) & $3(9.0)$ & $1(3.0)$ \\
Mean score & 77.2 & 88.3 \\
\hline
\end{tabular}

p-value $>0.05$.

Values are presented as number (\%)

statistical analysis, patient demographic data were analyzed using the independent t-test and the Mann-Whitney test (Table 1). The Lachman test results, KT-2000 arthrometer measurements, pivot shift test results, Lysholm scores, and IKDC scores were analyzed using the chi-square test (Tables 2-6). A p-value of $<0.05$ was considered statistically significant. The Lachman test, pivot shift test, and femoral tunnel obliquity measurement were conducted by an orthopedic surgeon who had $\geq 20$ years of experience in knee surgery and performed the surgery described above.

\section{Results}

\section{Knee Joint Stability}

In the control group, the preoperative Lachman test results were grade I in 2 patients (6\%), grade II in 21 patients (64\%), and grade III in 10 patients (30\%). In the experimental group, the preoperative Lachman test results were grade I in 1 patient $(3 \%)$, grade II in 23 patients (70\%), and grade III in 9 patients (27\%). At the last follow-up, remarkable improvements were observed in both groups; however, statistically significant differences between the groups were not found, with grade 0 in 27 patients (82\%), grade I in 3 patients (9\%), grade II in 2 patients (6\%), and grade III in 1 patient (3.0\%) in the control group, compared to grade 0 in 28 patients (85\%), grade I in 4 patients (12\%), and grade II in 1 patient (3.0\%) in the experimental group ( $>>0.05)$ (Table 2).

The mean side-to-side difference in anterior translation assessed
Table 6. International Knee Documentation Committee (IKDC) Score

\begin{tabular}{lcc}
\hline IKDC score & Trans tibia & Far anteromedial \\
\hline Normal & $21(68.0)$ & $23(69.7)$ \\
Nearly normal & $10(30.3)$ & $9(27.3)$ \\
Abnormal & $2(6.0)$ & $1(3.0)$ \\
Severely abnormal & $0(0.0)$ & $0(0.0)$ \\
\hline
\end{tabular}

p-value $>0.05$.

Values are presented as number (\%).

using the KT-2000 arthrometer was decreased in both groups between the preoperative and the last follow-up examination. The change was from $6.5 \mathrm{~mm}$ (range, $3-13 \mathrm{~mm}$ ) to $3.1 \mathrm{~mm}$ in the control group and from $6.37 \mathrm{~mm}$ (range, $3-12 \mathrm{~mm}$ ) to $2.8 \mathrm{~mm}$ in the experimental group, but no statistically significant intergroup difference was observed ( $p>0.05$ ) (Table 3 ).

Results of the pivot shift test improved between the preoperative and the last follow-up examinations in both groups. In the control group, the preoperative results were grade I in 9 patients (27\%), grade II in 16 patients (52\%), and grade III in 8 patients (24\%). In the experimental group, the preoperative results were grade I in 7 patients (21\%), grade II in 17 patients (52\%), and grade III in 9 patients (27\%). At the last follow-up, the results were grade 0 in 26 patients (78.8\%), grade I in 5 patients (15.2\%), and grade II in 2 patients (6\%) in the control group, and grade 0 in 30 patients (90.9\%), grade I in 2 patients (6.0\%), and grade II in 1 patient (3.0\%) in the experimental group. A statistically significant intergroup difference was observed in the postoperative pivot shift test results: the results were negative in 30 patients (90.9\%) in the experimental group and in 26 patients $(78.8 \%)$ in the control group $(\mathrm{p}=0.04)$ (Table 4$)$.

\section{Clinical Results}

The mean Lysholm score in the control group was 45.3 preoperatively and 86.2 at the last follow-up. There were 3 good cases (70-84 points, $9 \%$ ), 14 fair cases (55-69 points, $42 \%$ ), and 16 poor cases (0-54 points, $48 \%$ ) preoperatively, and 16 excellent cases (85-100 points, $49 \%), 10$ good cases (30.3\%), 4 fair cases (12.1\%), and 3 poor cases (9\%) at the last follow-up. The mean value in the experimental group was 45.8 preoperatively and 86.4 at the last follow-up. There were 3 good cases (70-84 points, 9\%), 14 fair cases (55-69 points, $42 \%$ ), and 16 poor cases (0-54 points, 48\%) preoperatively, and 19 excellent cases (85-100 points, $57.6 \%), 12$ good cases (36.4\%), 1 fair case (3.0\%), and 1 poor case (3.0\%) at the last follow-up. Thus, the Lysholm score was higher in the experimental group, but there was no significant difference between the groups ( $p>0.05$ ) (Table 5). The IKDC score showed 

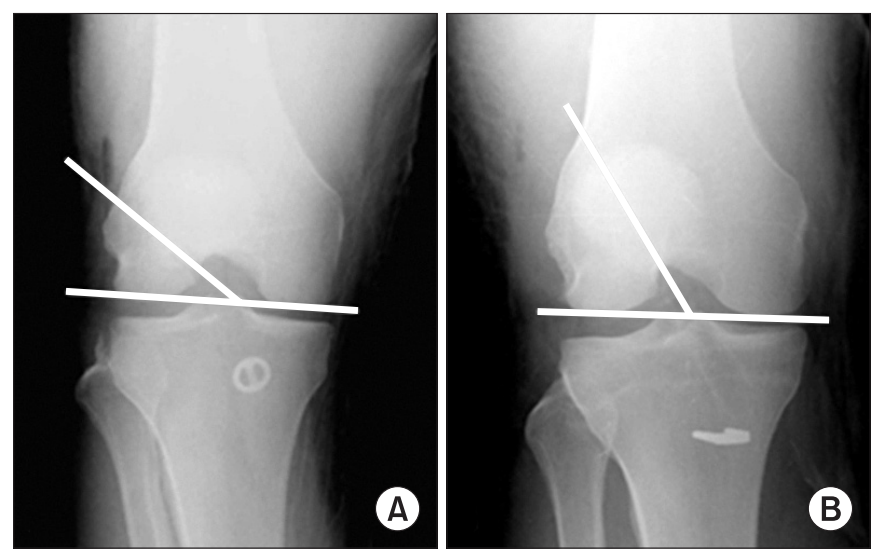

Fig. 4. Average femoral tunnel angle $31^{\circ}$ in 2 anteromedial portals (A) and $59^{\circ}$ in trans tibia (B).

no statistically significant difference between the groups ( $\mathrm{p}>0.05)$. In the control group, there were 22 abnormal (67\%) and 11 severely abnormal cases (33\%) preoperatively, and 21 normal (68\%), 10 nearly normal (30.3\%), and 2 abnormal cases (6.0\%) at the last follow-up. In the experimental group, there were 23 abnormal (70\%) and 10 severely abnormal cases (30\%) preoperatively, and 23 normal (69.7\%), 9 nearly normal (27.3\%), and 1 abnormal case (3.0\%) at the last follow-up (Table 6).

\section{Radiological Results (Femoral Tunnel Obliquity)}

The postoperative femoral tunnel obliquity was measured on the knee tunnel views obtained at the last follow-up. The mean value was significantly different between the groups, with $59^{\circ}$ in the control group and $31^{\circ}$ in the experimental group $(\mathrm{p}=0.03)$ (Fig. 4).

\section{Discussion}

The ACL can be divided into anteromedial and posterolateral bundles according to the attachment site on the tibia. These 2 bundles contribute to the stability of the knee: the anteromedial bundle provides anterior stability and the posterolateral bundle provides rotational stability ${ }^{10)}$. Zantop et al. ${ }^{11)}$ reported that the 2 bundles interact with each other for knee stability in response to the anteroposterior and combined rotational loads. For ACL tears, single-bundle reconstruction has been advocated as the treatment of choice with high success rates ${ }^{6,12)}$. However, some recent studies have shown that the procedure is not sufficient to restore intact knee rotational stability because of its inability to bring about biomechanical restoration of the posterolateral bundle $e^{6}$.
Recent studies have suggested methods to reduce residual rotational instability after single-bundle reconstruction. Because it has been reported that femoral tunnel obliquity is crucial for the recovery of rotational stability after the procedure, the focus has been set on lowering the reconstructed ligament's obliquity $^{13,14)}$. In the study of Jepsen et al. ${ }^{7)}$, patients' subjective satisfaction was significantly improved, and accordingly, greater clinical improvement could be expected when the femoral tunnel position was moved from the 11 oclock position to the 10 o'clock position. Scopp et al. ${ }^{15)}$ reported that the 10 or 2 oclock femoral tunnel position on the coronal plane was associated with the recovery of rotational stability, while they had no influence on the anteroposterior stability. Woo et al. ${ }^{16)}$ suggested that 10 or 2 oclock femoral tunnel positioning or double-bundle reconstruction techniques would be helpful for overcoming the limitation of single-bundle ACL reconstruction. The transtibial femoral tunnel drilling technique may not be effective for lowering the femoral tunnel obliquity because the obliquity of a femoral tunnel is determined according to that of a tibial tunnel. Brophy et al. ${ }^{17)}$ reported that restoring the anatomic footprint and ligament orientation to the pre-injury level resulted in improvements in the stability and biomechanics of the reconstructed knee. In the cadaveric study of Yagi et al. ${ }^{5}$, the kinematics of the reconstructed knee was closer to that of the intact knee when anatomic reconstruction was achieved.

In an attempt to lower the femoral tunnel obliquity using the transtibial technique, Rue et al. ${ }^{18)}$ moved the tibial tunnel starting point more proximally and medially compared to the conventional method and performed 10 or 2 oclock femoral tunnel placement. However, the limitations of their method include that the medial collateral ligament and pes anserinus can be damaged, and it is difficult to secure firm fixation to the tibia due to the short tibial tunnel length.

Anatomic ACL reconstruction techniques have been documented in many recent studies. Harner and Poehling ${ }^{9)}$ reported that femoral tunnel placement using an anteromedial portal, compared to that using a transtibial technique, is advantageous for locating more anatomically correct ACL attachment sites on the femur and lowering femoral tunnel obliquity, which makes femoral notchplasty unnecessary. However, this technique carries potential risks of short femoral tunnel creation, and damage to the articular cartilage of the medial femoral condyle, posterior wall of the lateral femoral condyle, and posterolateral structures such as the fibular nerve. Additionally, the knee should be flexed $\geq 90^{\circ}$ during tunnel drilling to avoid the complications, which could limit the field of view during surgery ${ }^{11)}$. 


\section{Kim et al. Anatomic Single Bundle Anterior Cruciate Ligament Reconstruction}

In this study, we suggested a new method using 2 anteromedial portals; a far anteromedial portal and the conventional anteromedial portal. The advantages of our method are as follows: 1) close replication of native femoral attachment of the ACL through visualization of the medial aspect of the lateral femoral condyle with the knee in hyperflexion; 2) shortened operation time; and 3) reduced complications such as posterior cortical bone loss.

Studies, including the current study, have shown that ACL reconstruction using a medial portal allows for anatomic reconstruction of the ACL and is effective for restoring anterior stability and rotational stability. However, in spite of the improvement in rotational stability, the clinical results were not significantly different between the experimental group and the control group. We attribute this to the relatively short followup period of 2-years. Indeed, some authors have suggested that statistical significance of clinical results can be better assessed with a long-term follow-up. Therefore, we think the patients should be followed for a longer period to investigate the relationship between the improvement in rotational stability and clinical results.

In ACL reconstruction using 2 anteromedial portals, the 2 portals should be placed at maximum distance from each other to prevent interference between the arthroscope and the reamer. In addition, care should be taken not to damage the articular cartilage of the medial femoral condyle during femoral tunnel drilling through the far anteromedial portal.

There are some limitations of our study. First, the patients in the control group were selected intentionally as those who were similar in age to those in the experimental group. However, this was an attempt to reduce the influence of age on knee joint function. Second, the obliquity of the reconstructed ligament was assessed using radiography rather than arthroscopy. However, arthroscopy is not conducive to the objective measurement of obliquity. In addition, considering that our purpose was to compare the obliquity between the 2 groups, and not to obtain the actual value, the use of radiography did not have an influence on the study results.

\section{Conclusions}

ACL reconstruction using 2 anteromedial portals can be effective for reproducing the anatomy of the ACL and obtaining good clinical results, because the technique allows for a better field of view and lower obliquity of the reconstructed ACL compared to the transtibial technique.

\section{References}

1. Buss DD, Warren RF, Wickiewicz TL, Galinat BJ, Panariello R. Arthroscopically assisted reconstruction of the anterior cruciate ligament with use of autogenous patellar-ligament grafts. Results after twenty-four to forty-two months. J Bone Joint Surg Am. 1993;75:1346-55.

2. Feller JA, Webster KE. A randomized comparison of patellar tendon and hamstring tendon anterior cruciate ligament reconstruction. Am J Sports Med. 2003;31:564-73.

3. Seon JK, Park SJ, Lee KB, Yoon TR, Seo HY, Song EK. Stability comparison of anterior cruciate ligament between double- and single-bundle reconstructions. Int Orthop. 2009;33:425-9.

4. Rosenberg TD, Franklin JL, Baldwin GN, Nelson KA. Extensor mechanism function after patellar tendon graft harvest for anterior cruciate ligament reconstruction. Am J Sports Med. 1992;20:519-25.

5. Yagi M, Wong EK, Kanamori A, Debski RE, Fu FH, Woo SL. Biomechanical analysis of an anatomic anterior cruciate ligament reconstruction. Am J Sports Med. 2002;30:660-6.

6. Aglietti P, Giron F, Cuomo P, Losco M, Mondanelli N. Singleand double-incision double-bundle ACL reconstruction. Clin Orthop Relat Res. 2007;454:108-13.

7. Jepsen CF, Lundberg-Jensen AK, Faunoe P. Does the position of the femoral tunnel affect the laxity or clinical outcome of the anterior cruciate ligament-reconstructed knee? A clinical, prospective, randomized, double-blind study. Arthroscopy. 2007;23:1326-33.

8. Kanamori A, Zeminski J, Rudy TW, Li G, Fu FH, Woo SL. The effect of axial tibial torque on the function of the anterior cruciate ligament: a biomechanical study of a simulated pivot shift test. Arthroscopy. 2002;18:394-8.

9. Harner CD, Poehling GG. Double bundle or double trouble? Arthroscopy. 2004;20:1013-4.

10. Amis AA, Dawkins GP. Functional anatomy of the anterior cruciate ligament. Fibre bundle actions related to ligament replacements and injuries. J Bone Joint Surg Br. 1991;73:2607.

11. Zantop T, Herbort M, Raschke MJ, Fu FH, Petersen W. The role of the anteromedial and posterolateral bundles of the anterior cruciate ligament in anterior tibial translation and internal rotation. Am J Sports Med. 2007;35:223-7.

12. Samuelson TS, Drez D Jr, Maletis GB. Anterior cruciate ligament graft rotation. Reproduction of normal graft rotation. Am J Sports Med. 1996;24:67-71. 
13. Loh JC, Fukuda Y, Tsuda E, Steadman RJ, Fu FH, Woo SL. Knee stability and graft function following anterior cruciate ligament reconstruction: Comparison between 11 o'clock and 10 o'clock femoral tunnel placement. 2002 Richard O'Connor Award paper. Arthroscopy. 2003;19:297-304.

14. O'Neill DB. Arthroscopically assisted reconstruction of the anterior cruciate ligament. A prospective randomized analysis of three techniques. J Bone Joint Surg Am. 1996;78:803-13.

15. Scopp JM, Jasper LE, Belkoff SM, Moorman CT 3rd. The effect of oblique femoral tunnel placement on rotational constraint of the knee reconstructed using patellar tendon autografts. Arthroscopy. 2004;20:294-9.

16. Woo SL, Kanamori A, Zeminski J, Yagi M, Papageorgiou C, $\mathrm{Fu}$ FH. The effectiveness of reconstruction of the anterior cruciate ligament with hamstrings and patellar tendon . A cadaveric study comparing anterior tibial and rotational loads. J Bone Joint Surg Am. 2002;84:907-14.

17. Brophy RH, Voos JE, Shannon FJ, Granchi CC, Wickiewicz TL, Warren RF, Pearle AD. Changes in the length of virtual anterior cruciate ligament fibers during stability testing: a comparison of conventional single-bundle reconstruction and native anterior cruciate ligament. Am J Sports Med. 2008;36:2196-203.

18. Rue JP, Ghodadra N, Bach BR Jr. Femoral tunnel placement in single-bundle anterior cruciate ligament reconstruction: a cadaveric study relating transtibial lateralized femoral tunnel position to the anteromedial and posterolateral bundle femoral origins of the anterior cruciate ligament. Am J Sports Med. 2008;36:73-9. 